\title{
On Geometric Circulant Matrices Whose Entries are Bi-Periodic Fibonacci and Bi-Periodic Lucas Numbers
}

\author{
Emrah Polatlı ${ }^{1}$ \\ ${ }^{1}$ Department of Mathematics, Faculty of Science and Arts, Bülent Ecevit University, Zonguldak, Turkey
}

\section{Article Info}

Keywords: Bi-periodic Fibonacci numbers, Bi-periodic Lucas numbers, Geometric circulant matrices, Spectral norm.

2010 AMS: 15A60, 11B39, $15 B 05$.

Received: 2 February 2020

Accepted: 13 July 2020

Available online: 29 September 2020

\begin{abstract}
In this study, we obtain upper and lower bounds for the spectral norms of the geometric circulant matrices with the bi-periodic Fibonacci numbers and bi-periodic Lucas numbers, respectively. Then we give some bounds for the spectral norms of Kronecker and Hadamard products of these matrices.
\end{abstract}

\section{Introduction}

The well-known Fibonacci and Lucas sequences are given by the following recursive equations: for $n \geq 0$,

$$
F_{0}=0, F_{1}=1, \quad F_{n+2}=F_{n+1}+F_{n}
$$

and

$$
L_{0}=2, L_{1}=1, \quad L_{n+2}=L_{n+1}+L_{n},
$$

respectively.

Many researchers gave various generalizations of the Fibonacci sequence in past fifty years. An interesting one, called bi-periodic Fibonacci sequence, was introduced by Edson and Yayenie in [5] as follows:

$$
q_{0}=0, q_{1}=1, \text { and } q_{n}=\left\{\begin{array}{ll}
a q_{n-1}+q_{n-2}, & \text { if } n \text { is even; } \\
b q_{n-1}+q_{n-2}, & \text { if } n \text { is odd. }
\end{array} \quad(n \geq 2),\right.
$$

where $a$ and $b$ are nonzero real numbers. They obtained many identities for the sequence $\left\{q_{n}\right\}_{n=0}^{\infty}$. For instance, they gave the following extended Binet formula

$$
q_{n}=\left(\frac{a^{1-\xi(n)}}{(a b)^{\left\lfloor\frac{n}{2}\right\rfloor}}\right) \frac{\alpha^{n}-\beta^{n}}{\alpha-\beta}, \quad(n \geq 0)
$$

where $\alpha=\left(a b+\sqrt{a^{2} b^{2}+4 a b}\right) / 2$ and $\beta=\left(a b-\sqrt{a^{2} b^{2}+4 a b}\right) / 2$. Here, $\xi(n)=n-2\left\lfloor\frac{n}{2}\right\rfloor$ is the parity function. In [4], Bilgici gave a general form of the Lucas sequence similar to the generalized Fibonacci sequence $\left\{q_{n}\right\}_{n=0}^{\infty}$ as follows:

$$
l_{0}=2, l_{1}=a, \text { and } l_{n}=\left\{\begin{array}{ll}
a l_{n-1}+l_{n-2}, & \text { if } n \text { is odd; } \\
b l_{n-1}+l_{n-2}, & \text { if } n \text { is even. }
\end{array} \quad(n \geq 2),\right.
$$


where $a$ and $b$ are nonzero real numbers. He also derived many identities for the sequence $\left\{l_{n}\right\}_{n=0}^{\infty}$. For example, he gave the following extended Binet formula

$$
l_{n}=\left(\frac{a^{\xi(n)}}{(a b)^{\left\lfloor\frac{n+1}{2}\right\rfloor}}\right)\left(\alpha^{n}+\beta^{n}\right), \quad(n \geq 0) .
$$

The $n \times n \quad r$-circulant matrix, $C_{r}$, associated with the numbers $c_{0}, c_{1}, \ldots, c_{n-1}$ is of the form

$$
c_{i j}= \begin{cases}c_{j-i}, & j \geq i \\ r c_{n+j-i}, & j<i\end{cases}
$$

that is

$$
C_{r}=\left(\begin{array}{cccccc}
c_{0} & c_{1} & c_{2} & \cdots & c_{n-2} & c_{n-1} \\
r c_{n-1} & c_{0} & c_{1} & \cdots & c_{n-3} & c_{n-2} \\
r c_{n-2} & r c_{n-1} & c_{0} & \cdots & c_{n-4} & c_{n-3} \\
\vdots & \vdots & \vdots & \ddots & \vdots & \vdots \\
r c_{2} & r c_{3} & r c_{4} & \cdots & c_{0} & c_{1} \\
r c_{1} & r c_{2} & r c_{3} & \cdots & r c_{n-1} & c_{0}
\end{array}\right)
$$

For $r=1$, the $r$-circulant matrix $C_{r}$ reduces to circulant matrix $C$, i.e.,

$$
C=\left(\begin{array}{cccccc}
c_{0} & c_{1} & c_{2} & \cdots & c_{n-2} & c_{n-1} \\
c_{n-1} & c_{0} & c_{1} & \cdots & c_{n-3} & c_{n-2} \\
c_{n-2} & c_{n-1} & c_{0} & \cdots & c_{n-4} & c_{n-3} \\
\vdots & \vdots & \vdots & \ddots & \vdots & \vdots \\
c_{2} & c_{3} & c_{4} & \cdots & c_{0} & c_{1} \\
c_{1} & c_{2} & c_{3} & \cdots & c_{n-1} & c_{0}
\end{array}\right)
$$

Circulant matrices, $r$-circulant matrices, and their versions have been studied in many papers. For example, in [20], Solak found some bounds for the spectral norms of circulant matrices with the Fibonacci and Lucas number entries. Afterwards, Shen and Cen [16] developed Solak's results. Later, many researchers studied different types of these matrices. For more details, we refer the interested reader to $[1-3,6,8,9,12,15,17-19,21-23,25]$.

In [10], Kızılateş and Tuğlu defined the $n \times n$ geometric circulant matrix, $C_{s^{*}}$, associated with the numbers $c_{0}, c_{1}, \ldots, c_{n-1}$ as

$$
C_{s^{*}}=\left(\begin{array}{cccccc}
c_{0} & c_{1} & c_{2} & \cdots & c_{n-2} & c_{n-1} \\
s c_{n-1} & c_{0} & c_{1} & \cdots & c_{n-3} & c_{n-2} \\
s^{2} c_{n-2} & s c_{n-1} & c_{0} & \cdots & c_{n-4} & c_{n-3} \\
\vdots & \vdots & \vdots & \ddots & \vdots & \vdots \\
s^{n-2} c_{2} & s^{n-3} c_{3} & s^{n-4} c_{4} & \cdots & c_{0} & c_{1} \\
s^{n-1} c_{1} & s^{n-2} c_{2} & s^{n-3} c_{3} & \cdots & s c_{n-1} & c_{0}
\end{array}\right) .
$$

They calculated bounds for the spectral norms of geometric circulant matrices with the generalized Fibonacci numbers and hyperharmonic Fibonacci numbers. Same authors [11] also found the norms of geometric and symmetric geometric circulant matrices with the Tribonacci numbers. In [13], Köme and Yazlık presented some bounds for the spectral norms of the $r$-circulant matrices with the bi-periodic Fibonacci and Lucas numbers.

The purpose of this paper is to find some new upper and lower bounds for the spectral norms of the geometric circulant matrices with the bi-periodic Fibonacci numbers and bi-periodic Lucas numbers, respectively.

Now we need the following definitions and lemmas to derive new bounds.

The Euclidean (Frobenius) norm of matrix $A\left(A=\left(a_{i j}\right)\right.$ be any $m \times n$ matrix $)$ is defined as

$$
\|A\|_{E}=\sqrt{\sum_{i=1}^{m} \sum_{j=1}^{n}\left|a_{i j}\right|^{2}} .
$$

Let $A^{H}$ is conjugate transpose of the matrix $A$. Then the spectral norm of matrix $A$ is defined as

$$
\|A\|_{2}=\sqrt{\max _{1 \leq i \leq n} \lambda_{i}\left(A^{H} A\right)}
$$

The following inequality [7] holds:

$$
\frac{1}{\sqrt{n}}\|A\|_{E} \leq\|A\|_{2} \leq\|A\|_{E}
$$

Lemma 1.1 ( [7]). Let $A=\left(a_{i j}\right)$ and $B=\left(b_{i j}\right)$ be any $m \times n$ matrices and let $A \circ B$ is the Hadamard product of $A$ and $B$. Then

$$
\|A \circ B\|_{2} \leq\|A\|_{2}\|B\|_{2} .
$$


Lemma 1.2 ( [14]). Let $A=\left(a_{i j}\right)$ and $B=\left(b_{i j}\right)$ be any $m \times n$ matrices. Then

$$
\|A \circ B\|_{2} \leq r_{1}(A) c_{1}(B)
$$

where

$$
r_{1}(A)=\max _{1 \leq i \leq m} \sqrt{\sum_{j=1}^{n}\left|a_{i j}\right|^{2}} \text { and } \quad c_{1}(B)=\max _{1 \leq j \leq n} \sqrt{\sum_{i=1}^{m}\left|b_{i j}\right|^{2}} .
$$

Lemma 1.3 ( [7]). Let $A=\left(a_{i j}\right)$ and $B=\left(b_{i j}\right)$ be any $m \times n$ matrices and let $A \otimes B$ is the Kronecker product of $A$ and $B$. Then $\|A \otimes B\|_{2}=\|A\|_{2}\|B\|_{2}$.

\section{Main Results}

Theorem 2.1. Let $F=C_{S^{*}}\left(\left(\frac{a}{b}\right)^{\frac{\xi(0)}{2}} q_{0},\left(\frac{a}{b}\right)^{\frac{\xi(1)}{2}} q_{1}, \ldots,\left(\frac{a}{b}\right)^{\frac{\xi(n-1)}{2}} q_{n-1}\right)$ be an $n \times n$ geometric circulant matrix where a and $b$ are nonzero positive real numbers and $s \in \mathbb{C}$. Then

(i) If $|s|>1$, we have

$$
\sqrt{\frac{q_{n-1} q_{n}}{b}} \leq\|F\|_{2} \leq|s| \sqrt{\left(\frac{|s|^{2 n-2}-1}{|s|^{2}-1}\right) \frac{q_{n-1} q_{n}}{b}}
$$

(ii) If $|s|<1$, we have

$$
\frac{|s| \sqrt{a b}}{b \sqrt{a b+4}} \sqrt{\frac{2|s|^{2 n+2}-|s|^{2 n}(a b+2)-|s|^{2} l_{2 n}+l_{2 n-2}}{|s|^{4}-|s|^{2}(a b+2)+1}-2\left(\frac{|s|^{2 n}-(-1)^{n}}{|s|^{2}+1}\right)} \leq\|F\|_{2} \leq \sqrt{\frac{(n-1) q_{n-1} q_{n}}{b}} .
$$

Proof. If we consider the definition of $F=C_{S^{*}}\left(\left(\frac{a}{b}\right)^{\frac{\xi(0)}{2}} q_{0},\left(\frac{a}{b}\right)^{\frac{\xi(1)}{2}} q_{1}, \ldots,\left(\frac{a}{b}\right)^{\frac{\xi(n-1)}{2}} q_{n-1}\right)$, then we have the following matrix:

$$
F=\left(\begin{array}{ccccc}
\left(\frac{a}{b}\right)^{\frac{\xi(0)}{2}} q_{0} & \left(\frac{a}{b}\right)^{\frac{\xi(1)}{2}} q_{1} & \left(\frac{a}{b}\right)^{\frac{\xi(2)}{2}} q_{2} & \cdots & \left(\frac{a}{b}\right)^{\frac{\xi(n-1)}{2}} q_{n-1} \\
s\left(\frac{a}{b}\right)^{\frac{\xi(n-1)}{2}} q_{n-1} & \left(\frac{a}{b}\right)^{\frac{\xi(0)}{2}} q_{0} & \left(\frac{a}{b}\right)^{\frac{\xi(1)}{2}} q_{1} & \cdots & \left(\frac{a}{b}\right)^{\frac{\xi(n-2)}{2}} q_{n-2} \\
s^{2}\left(\frac{a}{b}\right)^{\frac{\xi(n-2)}{2}} q_{n-2} & s\left(\frac{a}{b}\right)^{\frac{\xi(n-1)}{2}} q_{n-1} & \left(\frac{a}{b}\right)^{\frac{\xi(0)}{2}} q_{0} & \cdots & \left(\frac{a}{b}\right)^{\frac{\xi(n-3)}{2}} q_{n-3} \\
\vdots & \vdots & \vdots & \ddots & \vdots \\
s^{n-1}\left(\frac{a}{b}\right)^{\frac{\xi(1)}{2}} q_{1} & s^{n-2}\left(\frac{a}{b}\right)^{\frac{\xi(2)}{2}} q_{2} & s^{n-3}\left(\frac{a}{b}\right)^{\frac{\xi(3)}{2}} q_{3} & \cdots & \left(\frac{a}{b}\right)^{\frac{\xi(0)}{2}} q_{0}
\end{array}\right)
$$

Thus we get the Euclidean norm of the matrix $F$ as

$$
\|F\|_{E}^{2}=\sum_{k=0}^{n-1}(n-k)\left(\frac{a}{b}\right)^{\xi(k)} q_{k}^{2}+\sum_{k=1}^{n-1} k\left|s^{n-k}\right|^{2}\left(\frac{a}{b}\right)^{\xi(k)} q_{k}^{2} .
$$

(i) If $|s|>1$, from [24, Theorem 2.3], we obtain

$$
\begin{aligned}
\|F\|_{E}^{2} & \geq \sum_{k=0}^{n-1}(n-k)\left(\frac{a}{b}\right)^{\xi(k)} q_{k}^{2}+\sum_{k=1}^{n-1} k\left(\frac{a}{b}\right)^{\xi(k)} q_{k}^{2} \\
& =n \sum_{k=0}^{n-1}\left(\frac{a}{b}\right)^{\xi(k)} q_{k}^{2} \\
& =n \frac{q_{n-1} q_{n}}{b} .
\end{aligned}
$$

So we have

$$
\frac{1}{\sqrt{n}}\|F\|_{E} \geq \sqrt{\frac{q_{n-1} q_{n}}{b}}
$$

that is

$$
\sqrt{\frac{q_{n-1} q_{n}}{b}} \leq\|F\|_{2}
$$


Now, let us choose the matrices

$$
A=\left(\begin{array}{cccccc}
q_{0} & 1 & 1 & \cdots & 1 & 1 \\
s & q_{0} & 1 & \cdots & 1 & 1 \\
s^{2} & s & q_{0} & \cdots & 1 & 1 \\
\vdots & \vdots & \vdots & \ddots & \vdots & \vdots \\
s^{n-1} & s^{n-2} & s^{n-3} & \cdots & s & q_{0}
\end{array}\right)
$$

and

$$
B=\left(\begin{array}{ccccc}
\left(\frac{a}{b}\right)^{\frac{\xi(0)}{2}} q_{0} & \left(\frac{a}{b}\right)^{\frac{\xi(1)}{2}} q_{1} & \left(\frac{a}{b}\right)^{\frac{\xi(2)}{2}} q_{2} & \cdots & \left(\frac{a}{b}\right)^{\frac{\xi(n-1)}{2}} q_{n-1} \\
\left(\frac{a}{b}\right)^{\frac{\xi(n-1)}{2}} q_{n-1} & \left(\frac{a}{b}\right)^{\frac{\xi(0)}{2}} q_{0} & \left(\frac{a}{b}\right)^{\frac{\xi(1)}{2}} q_{1} & \cdots & \left(\frac{a}{b}\right)^{\frac{\xi(n-2)}{2}} q_{n-2} \\
\left(\frac{a}{b}\right)^{\frac{\xi(2)}{2}} q_{2} & \left(\frac{a}{b}\right)^{\frac{\xi(n-1)}{2}} q_{n-1} & \left(\frac{a}{b}\right)^{\frac{\xi(0)}{2}} q_{0} & \cdots & \left(\frac{a}{b}\right)^{\frac{\xi(n-3)}{2}} q_{n-3} \\
\vdots & \vdots & \vdots & \ddots & \vdots \\
\left(\frac{a}{b}\right)^{\frac{\xi(1)}{2}} q_{1} & \left(\frac{a}{b}\right)^{\frac{\xi(2)}{2}} q_{2} & \left(\frac{a}{b}\right)^{\frac{\xi(3)}{2}} q_{3} & \cdots & \left(\frac{a}{b}\right)^{\frac{\xi(0)}{2}} q_{0}
\end{array}\right)
$$

such that $F=A \circ B$. Therefore we obtain

$$
r_{1}(A)=\max _{1 \leq i \leq n} \sqrt{\sum_{j=1}^{n}\left|a_{i j}\right|^{2}}=\sqrt{q_{0}^{2}+\sum_{k=1}^{n-1}|s|^{2 k}}=|s| \sqrt{\frac{|s|^{2 n-2}-1}{|s|^{2}-1}}
$$

and

$$
c_{1}(B)=\max _{1 \leq j \leq n} \sqrt{\sum_{i=1}^{n}\left|b_{i j}\right|^{2}}=\sqrt{\sum_{k=0}^{n-1}\left(\frac{a}{b}\right)^{\xi(k)} q_{k}^{2}}=\sqrt{\frac{q_{n-1} q_{n}}{b}}
$$

By Lemma 1.2, we get

$$
\sqrt{\frac{q_{n-1} q_{n}}{b}} \leq\|F\|_{2} \leq|s| \sqrt{\left(\frac{|s|^{2 n-2}-1}{|s|^{2}-1}\right) \frac{q_{n-1} q_{n}}{b}} .
$$

(ii) If $|s|<1$, then we have

$$
\begin{aligned}
\|F\|_{E}^{2} & \geq \sum_{k=0}^{n-1}(n-k)\left|s^{n-k}\right|^{2}\left(\frac{a}{b}\right)^{\xi(k)} q_{k}^{2}+\sum_{k=1}^{n-1} k\left|s^{n-k}\right|^{2}\left(\frac{a}{b}\right)^{\xi(k)} q_{k}^{2} \\
& =n|s|^{2 n} \sum_{k=0}^{n-1}\left(\frac{\left(\frac{a}{b}\right)^{\frac{\xi(k)}{2}} q_{k}}{|s|^{k}}\right)^{2} \\
& =\frac{a n|s|^{2 n}}{b(a b+4)}\left(\sum_{k=0}^{n-1}\left(\frac{\alpha^{2}}{|s|^{2} a b}\right)^{k}+\sum_{k=0}^{n-1}\left(\frac{\beta^{2}}{|s|^{2} a b}\right)^{k}-2 \sum_{k=0}^{n-1}\left(\frac{-1}{|s|^{2}}\right)^{k}\right) \\
& =\frac{a n|s|^{2}}{b(a b+4)}\left(\frac{2|s|^{2 n+2}-|s|^{2 n}(a b+2)-|s|^{2} l_{2 n}+l_{2 n-2}}{|s|^{4}-|s|^{2}(a b+2)+1}-2\left(\frac{|s|^{2 n}-(-1)^{n}}{|s|^{2}+1}\right)\right) .
\end{aligned}
$$

Therefore we obtain the following lower bound:

$$
\frac{|s| \sqrt{a b}}{b \sqrt{a b+4}} \sqrt{\frac{2|s|^{2 n+2}-|s|^{2 n}(a b+2)-|s|^{2} l_{2 n}+l_{2 n-2}}{|s|^{4}-|s|^{2}(a b+2)+1}-2\left(\frac{|s|^{2 n}-(-1)^{n}}{|s|^{2}+1}\right)} \leq\|F\|_{2} .
$$

In the meantime, let the matrices $A$ and $B$ be given in (2.1) and (2.2) such that $F=A \circ B$. Then we have

$$
r_{1}(A)=\max _{1 \leq i \leq n} \sqrt{\sum_{j=1}^{n}\left|a_{i j}\right|^{2}}=\sqrt{n-1}
$$

and

$$
c_{1}(B)=\max _{1 \leq j \leq n} \sqrt{\sum_{i=1}^{n}\left|b_{i j}\right|^{2}}=\sqrt{\sum_{k=0}^{n-1}\left(\frac{a}{b}\right)^{\xi(k)} q_{k}^{2}}=\sqrt{\frac{q_{n-1} q_{n}}{b}} .
$$

Combining the above equations, we get the following inequality:

$$
\frac{|s| \sqrt{a b}}{b \sqrt{a b+4}} \sqrt{\frac{2|s|^{2 n+2}-|s|^{2 n}(a b+2)-|s|^{2} l_{2 n}+l_{2 n-2}}{|s|^{4}-|s|^{2}(a b+2)+1}-2\left(\frac{|s|^{2 n}-(-1)^{n}}{|s|^{2}+1}\right)} \leq\|F\|_{2} \leq \sqrt{\frac{(n-1) q_{n-1} q_{n}}{b}} .
$$


Theorem 2.2. Let $L=C_{S^{*}}\left(\left(\frac{a}{b}\right)^{\frac{\xi(1)}{2}} l_{0},\left(\frac{a}{b}\right)^{\frac{\xi(2)}{2}} l_{1}, \ldots,\left(\frac{a}{b}\right)^{\frac{\xi(n)}{2}} l_{n-1}\right)$ be an $n \times n$ geometric circulant matrix where a and $b$ are nonzero positive real numbers and $s \in \mathbb{C}$. Then

(i) If $|s|>1$, we have

$$
\sqrt{\frac{l_{n-1} l_{n}+2 a}{b}} \leq\|L\|_{2} \leq \sqrt{\left(\frac{|s|^{2 n}-1}{|s|^{2}-1}\right) \frac{l_{n-1} l_{n}+2 a}{b}}
$$

(ii) If $|s|<1$, we have

$$
\frac{|s| \sqrt{a b}}{b} \sqrt{\frac{2|s|^{2 n+2}-|s|^{2 n}(a b+2)-|s|^{2} l_{2 n}+l_{2 n-2}}{|s|^{4}-|s|^{2}(a b+2)+1}+2\left(\frac{|s|^{2 n}-(-1)^{n}}{|s|^{2}+1}\right)} \leq\|L\|_{2} \leq \sqrt{\frac{n}{b}\left(l_{n-1} l_{n}+2 a\right)}
$$

Proof. Firstly, we have the following matrix:

$$
L=\left(\begin{array}{ccccc}
\left(\frac{a}{b}\right)^{\frac{\xi(1)}{2}} l_{0} & \left(\frac{a}{b}\right)^{\frac{\xi(2)}{2}} l_{1} & \left(\frac{a}{b}\right)^{\frac{\xi(3)}{2}} l_{2} & \cdots & \left(\frac{a}{b}\right)^{\frac{\xi(n)}{2}} l_{n-1} \\
s\left(\frac{a}{b}\right)^{\frac{\xi(n)}{2}} l_{n-1} & \left(\frac{a}{b}\right)^{\frac{\xi(1)}{2}} l_{0} & \left(\frac{a}{b}\right)^{\frac{\xi(2)}{2}} l_{1} & \cdots & \left(\frac{a}{b}\right)^{\frac{\xi(n-1)}{2}} l_{n-2} \\
s^{2}\left(\frac{a}{b}\right)^{\frac{\xi(n-1)}{2}} l_{n-2} & s\left(\frac{a}{b}\right)^{\frac{\xi(n)}{2}} l_{n-1} & \left(\frac{a}{b}\right)^{\frac{\xi(1)}{2}} l_{0} & \cdots & \left(\frac{a}{b}\right)^{\frac{\xi(n-2)}{2}} l_{n-3} \\
\vdots & \vdots & \vdots & \ddots & \vdots \\
s^{n-1}\left(\frac{a}{b}\right)^{\frac{\xi(2)}{2}} l_{1} & s^{n-2}\left(\frac{a}{b}\right)^{\frac{\xi(3)}{2}} l_{2} & s^{n-3}\left(\frac{a}{b}\right)^{\frac{\xi(4)}{2}} l_{3} & \cdots & \left(\frac{a}{b}\right)^{\frac{\xi(1)}{2}} l_{0}
\end{array}\right) .
$$

Thus we get the Euclidean norm of the matrix $L$ as

$$
\|L\|_{E}^{2}=\sum_{k=0}^{n-1}(n-k)\left(\frac{a}{b}\right)^{\xi(k+1)} l_{k}^{2}+\sum_{k=1}^{n-1} k\left|s^{n-k}\right|^{2}\left(\frac{a}{b}\right)^{\xi(k+1)} l_{k}^{2} .
$$

(i) If $|s|>1$, from [13, Theorem 2.1], we get

$$
\begin{aligned}
\|L\|_{E}^{2} & \geq \sum_{k=0}^{n-1}(n-k)\left(\frac{a}{b}\right)^{\xi(k+1)} l_{k}^{2}+\sum_{k=1}^{n-1} k\left(\frac{a}{b}\right)^{\xi(k+1)} l_{k}^{2} \\
& =n \sum_{k=0}^{n-1}\left(\frac{a}{b}\right)^{\xi(k+1)} l_{k}^{2} \\
& =\frac{n}{b}\left(l_{n-1} l_{n}+2 a\right) .
\end{aligned}
$$

So we obtain

$$
\frac{1}{\sqrt{n}}\|L\|_{E} \geq \sqrt{\frac{l_{n-1} l_{n}+2 a}{b}}
$$

that is

$$
\sqrt{\frac{l_{n-1} l_{n}+2 a}{b}} \leq\|L\|_{2} .
$$

In the meantime, let us choose the matrices

$$
C=\left(\begin{array}{cccccc}
1 & 1 & 1 & \cdots & 1 & 1 \\
s & 1 & 1 & \cdots & 1 & 1 \\
s^{2} & s & 1 & \cdots & 1 & 1 \\
\vdots & \vdots & \vdots & \ddots & \vdots & \vdots \\
s^{n-1} & s^{n-2} & s^{n-3} & \ldots & s & 1
\end{array}\right)
$$

and

$$
D=\left(\begin{array}{ccccc}
\left(\frac{a}{b}\right)^{\frac{\xi(1)}{2}} l_{0} & \left(\frac{a}{b}\right)^{\frac{\xi(2)}{2}} l_{1} & \left(\frac{a}{b}\right)^{\frac{\xi(3)}{2}} l_{2} & \cdots & \left(\frac{a}{b}\right)^{\frac{\xi(n)}{2}} l_{n-1} \\
\left(\frac{a}{b}\right)^{\frac{\xi(n)}{2}} l_{n-1} & \left(\frac{a}{b}\right)^{\frac{\xi(1)}{2}} l_{0} & \left(\frac{a}{b}\right)^{\frac{\xi(2)}{2}} q_{1} & \cdots & \left(\frac{a}{b}\right)^{\frac{\xi(n-1)}{2}} l_{n-2} \\
\left(\frac{a}{b}\right)^{\frac{\xi(3)}{2}} l_{2} & \left(\frac{a}{b}\right)^{\frac{\xi \xi(n)}{2}} l_{n-1} & \left(\frac{a}{b}\right)^{\frac{\xi(1)}{2}} l_{0} & \cdots & \left(\frac{a}{b}\right)^{\frac{\xi(n-2)}{2}} l_{n-3} \\
\vdots & \vdots & \vdots & \ddots & \vdots \\
\left(\frac{a}{b}\right)^{\frac{\xi(2)}{2}} l_{1} & \left(\frac{a}{b}\right)^{\frac{\xi}{2}} l_{2} & \left(\frac{a}{b}\right)^{\frac{\xi(4)}{2}} l_{3} & \cdots & \left(\frac{a}{b}\right)^{\frac{\xi(1)}{2}} l_{0}
\end{array}\right)
$$


such that $L=C \circ D$. Therefore we have

$$
r_{1}(C)=\max _{1 \leq i \leq n} \sqrt{\sum_{j=1}^{n}\left|c_{i j}\right|^{2}}=\sqrt{\sum_{k=0}^{n-1}|s|^{2 k}}=\sqrt{\frac{|s|^{2 n}-1}{|s|^{2}-1}}
$$

and

$$
c_{1}(D)=\max _{1 \leq j \leq n} \sqrt{\sum_{i=1}^{n}\left|d_{i j}\right|^{2}}=\sqrt{\sum_{k=0}^{n-1}\left(\frac{a}{b}\right)^{\xi(k+1)} l_{k}^{2}}=\sqrt{\frac{l_{n-1} l_{n}+2 a}{b}} .
$$

By Lemma 1.2, we obtain

$$
\sqrt{\frac{l_{n-1} l_{n}+2 a}{b}} \leq\|L\|_{2} \leq \sqrt{\left(\frac{|s|^{2 n}-1}{|s|^{2}-1}\right)\left(\frac{l_{n-1} l_{n}+2 a}{b}\right)}
$$

(ii) If $|s|<1$, then we get

$$
\begin{aligned}
\|L\|_{E}^{2} & \geq \sum_{k=0}^{n-1}(n-k)\left|s^{n-k}\right|^{2}\left(\frac{a}{b}\right)^{\xi(k+1)} l_{k}^{2}+\sum_{k=1}^{n-1} k\left|s^{n-k}\right|^{2}\left(\frac{a}{b}\right)^{\xi(k+1)} l_{k}^{2} \\
& =n|s|^{2 n} \sum_{k=0}^{n-1}\left(\frac{\left(\frac{a}{b}\right)^{\frac{\xi(k+1)}{2}} l_{k}}{|s|^{k}}\right)^{2} \\
& =\frac{a n|s|^{2 n}}{b}\left(\sum_{k=0}^{n-1}\left(\frac{\alpha^{2}}{|s|^{2} a b}\right)^{k}+\sum_{k=0}^{n-1}\left(\frac{\beta^{2}}{|s|^{2} a b}\right)^{k}+2 \sum_{k=0}^{n-1}\left(\frac{-1}{|s|^{2}}\right)^{k}\right) \\
& =\frac{a n|s|^{2}}{b}\left(\frac{2|s|^{2 n+2}-|s|^{2 n}(a b+2)-|s|^{2} l_{2 n}+l_{2 n-2}}{|s|^{4}-|s|^{2}(a b+2)+1}+2 \frac{|s|^{2 n}-(-1)^{n}}{|s|^{2}+1}\right) .
\end{aligned}
$$

Thus we get the following inequality:

$$
\frac{|s| \sqrt{a b}}{b} \sqrt{\frac{2|s|^{2 n+2}-|s|^{2 n}(a b+2)-|s|^{2} l_{2 n}+l_{2 n-2}}{|s|^{4}-|s|^{2}(a b+2)+1}+2\left(\frac{|s|^{2 n}-(-1)^{n}}{|s|^{2}+1}\right)} \leq\|L\|_{2} .
$$

In the meantime, let the matrices $C$ and $D$ be given in (2.3) and (2.4) such that $L=C \circ D$. Then we have

$$
r_{1}(C)=\max _{1 \leq i \leq n} \sqrt{\sum_{j=1}^{n}\left|c_{i j}\right|^{2}}=\sqrt{n}
$$

and

$$
c_{1}(D)=\max _{1 \leq j \leq n} \sqrt{\sum_{i=1}^{n}\left|d_{i j}\right|^{2}}=\sqrt{\sum_{k=0}^{n-1}\left(\frac{a}{b}\right)^{\xi(k+1)} l_{k}^{2}}=\sqrt{\frac{l_{n-1} l_{n}+2 a}{b}} .
$$

Combining the above equations, we have the following inequality:

$$
\frac{|s| \sqrt{a b}}{b} \sqrt{\frac{2|s|^{2 n+2}-|s|^{2 n}(a b+2)-|s|^{2} l_{2 n}+l_{2 n-2}}{|s|^{4}-|s|^{2}(a b+2)+1}+2\left(\frac{|s|^{2 n}-(-1)^{n}}{|s|^{2}+1}\right)} \leq\|L\|_{2} \leq \sqrt{\frac{n}{b}\left(l_{n-1} l_{n}+2 a\right)} .
$$

Corollary 2.3. Let $a$ and $b$ be nonzero positive real numbers and the matrices $F$ and $L$ be as in Theorem 2.1 and Theorem 2.2, respectively.

(i) If $|s|>1$, then we have

$$
\|F \circ L\|_{2} \leq \frac{|s|}{b\left(|s|^{2}-1\right)} \sqrt{\left(|s|^{2 n-2}-1\right)\left(|s|^{2 n}-1\right) q_{n-1} q_{n}\left(l_{n-1} l_{n}+2 a\right)} .
$$

(ii) If $|s|<1$, then we have

$$
\|F \circ L\|_{2} \leq \frac{1}{b} \sqrt{n(n-1) q_{n-1} q_{n}\left(l_{n-1} l_{n}+2 a\right)} .
$$

Proof. The proof follows from Lemma 1.1, Theorem 2.1 and Theorem 2.2.

Corollary 2.4. Let $a$ and $b$ be nonzero positive real numbers and the matrices $F$ and $L$ be as in Theorem 2.1 and Theorem 2.2, respectively. 
(i) If $|s|>1$, then we have

$$
\|F \otimes L\|_{2} \geq \frac{1}{b} \sqrt{q_{n-1} q_{n}\left(l_{n-1} l_{n}+2 a\right)}
$$

and

$$
\|F \otimes L\|_{2} \leq \frac{|s|}{b\left(|s|^{2}-1\right)} \sqrt{\left(|s|^{2 n-2}-1\right)\left(|s|^{2 n}-1\right) q_{n-1} q_{n}\left(l_{n-1} l_{n}+2 a\right)} .
$$

(ii) If $|s|<1$, then we have

$$
\|F \otimes L\|_{2} \geq \frac{a|s|^{2}}{b \sqrt{a b+4}} \sqrt{\left(\frac{2|s|^{2 n+2}-|s|^{2 n}(a b+2)-|s|^{2} l_{2 n}+l_{2 n-2}}{|s|^{4}-|s|^{2}(a b+2)+1}\right)^{2}-4\left(\frac{|s|^{2 n}-(-1)^{n}}{|s|^{2}+1}\right)^{2}}
$$

and

$$
\|F \otimes L\|_{2} \leq \frac{1}{b} \sqrt{n(n-1) q_{n-1} q_{n}\left(l_{n-1} l_{n}+2 a\right)} .
$$

Proof. The proof follows from Lemma 1.3, Theorem 2.1 and Theorem 2.2.

\section{References}

[1] M. Bahşi, S. Solak, On the norms of r-circulant matrices with the hyper-Fibonacci and Lucas numbers, J. Math. Inequal., 8(4) (2014) 693-705.

[2] M. Bahsi, On the norms of circulant matrices with the generalized Fibonacci and Lucas numbers, TWMS J. Pure Appl. Math., 6(1) (2015), 84-92.

[3] M. Bahsi, On the norms of $r$-circulant matrices with the hyperharmonic numbers, J. Math. Inequal., 10(2) (2016), 445-458.

[4] G. Bilgici, Two generalizations of Lucas sequence, Appl. Math. Comput., 245 (2014), 526-538.

[5] M. Edson, O. Yayenie, A new generalization of Fibonacci sequence and extended Binet's formula, INTEGERS, 9 (2009), 639-654.

[6] C. He, J. Ma, K. Zhang, Z. Wang, The upper bound estimation on the spectral norm of r-circulant matrices with the Fibonacci and Lucas numbers, J. Inequal. Appl., 2015 (2015), Article ID 72, 10 pages.

[7] R. Horn, C. Johnson, Topics in Matrix Analysis, Cambridge University Press, Cambridge, 1991.

[8] Z. Jiang, Z. Zhou, A note on spectral norms of even-order r-circulant matrices, Appl. Math. Comput., 250 (2015), $368-371$.

[9] C. Kizilates, On the Ouadra Lucas-Jacobsthal numbers, Karaelmas Fen ve Müh. Derg., 7(2) (2017), 619-621.

[10] C. Kızılateş, N. Tuğlu, On the bounds for the spectral norms of geometric circulant matrices, J. Inequal. Appl., 2016 (2016), Article ID 312,15 pages.

[11] C. Kızılateş, N. Tuğlu, On the Norms of Geometric and Symmetric Geometric Circulant Matrices with the Tribonacci Number, Gazi Univ. J. Sci., 31(2) (2018), 555-567.

[12] E. G. Koçer, T. Mansour, N. Tuğlu, Norms of circulant and semicirculant matrices with Horadams's numbers, Ars Comb., 85 (2007), 353-359.

[13] C. Köme, Y. Yazlık, On the spectral norms of r-circulant matrices with the biperiodic Fibonacci and Lucas numbers, J. Inequal. Appl., 2017 (2017), Article ID 192, 12 pages.

[14] R. Mathias, The spectral norm of a nonnegative matrix, Linear Algebra Appl., 139 (1990), 269-284.

[15] B. Radičić, On k-circulant matrices (with geometric sequence), Quaest. Math., 39 (2016), 135-144.

[16] S. Q. Shen, J. M. Cen, On the bounds for the norms of r-circulant matrices with Fibonacci and Lucas numbers, Appl. Math. Comput., 216 (2010), 2891-2897.

[17] S. Q. Shen, J. M. Cen, On the Spectral Norms of r-Circulant Matrices with the k-Fibonacci and k-Lucas Numbers, Int. J. Contemp. Math. Sci., 5(12) (2010), 569-578.

[18] B. Shi, The spectral norms of geometric circulant matrices with the generalized $k$-Horadam numbers, J. Inequal. Appl., 2018 (2018), Article ID 14, 9 pages.

[19] W. Sintunavarat, The upper bound estimation for the spectral norm of r-circulant and symmetric $r$-circulant matrices with the Padovan sequence, $\mathbf{J}$ Nonlinear Sci. Appl., 9 (2016), 92-101.

[20] S. Solak, On the norms of circulant matrices with the Fibonacci and Lucas numbers, Appl. Math. Comput., 160 (2005), 125-132.

[21] N. Tuğlu, C. Kızılateş, On the norms of circulant and r-circulant matrices with the hyperharmonic Fibonacci numbers, J. Inequal. Appl., 2015 (2015), Article ID 253, 11 pages.

[22] N. Tuğlu, C. Kızılateş, On the norms of some special matrices with the harmonic Fibonacci numbers, Gazi Univ. J. Sci., 28(3) (2015), 497-501.

[23] N. Tuğlu, C. Kızılateş, S. Kesim, On the harmonic and hyperharmonic Fibonacci numbers, Adv. Difference Equ., 2015 (2015), Article ID 297, 12 pages.

[24] O. Yayenie, New identities for generalized Fibonacci sequences and new generalization of Lucas sequences, SEA Bull. Math., 36 (2012), 739-752.

[25] Y. Yazlık, N. Taşkara, On the norms of an r-circulant matrix with the generalized k-Horadam numbers, J. Inequal. Appl., 2013 (2013), Article ID 394, 8 pages. 